\title{
Gradhiva
}

GRADHIV

Revue d'anthropologie et d'histoire des arts

17 | 2013

L'esthétique du geste technique

\section{Dessins chamaniques et espace virtuel dans le chamanisme khakasse}

Drums and virtual space in Khakas shamanism

\section{Charles Stépanoff}

\section{(2) OpenEdition \\ 1 Journals}

\section{Édition électronique}

URL : http://journals.openedition.org/gradhiva/2649

DOI : 10.4000/gradhiva.2649

ISSN : 1760-849X

Éditeur

Musée du quai Branly Jacques Chirac

\section{Édition imprimée}

Date de publication : 16 mai 2013

Pagination : 144-169

ISBN : 978-2-35744-049-74

ISSN : 0764-8928

\section{Référence électronique}

Charles Stépanoff, «Dessins chamaniques et espace virtuel dans le chamanisme khakasse », Gradhiva [En ligne], 17 | 2013, mis en ligne le 28 mai 2013, consulté le 10 décembre 2020. URL : http:// journals.openedition.org/gradhiva/2649; DOI : https://doi.org/10.4000/gradhiva.2649 


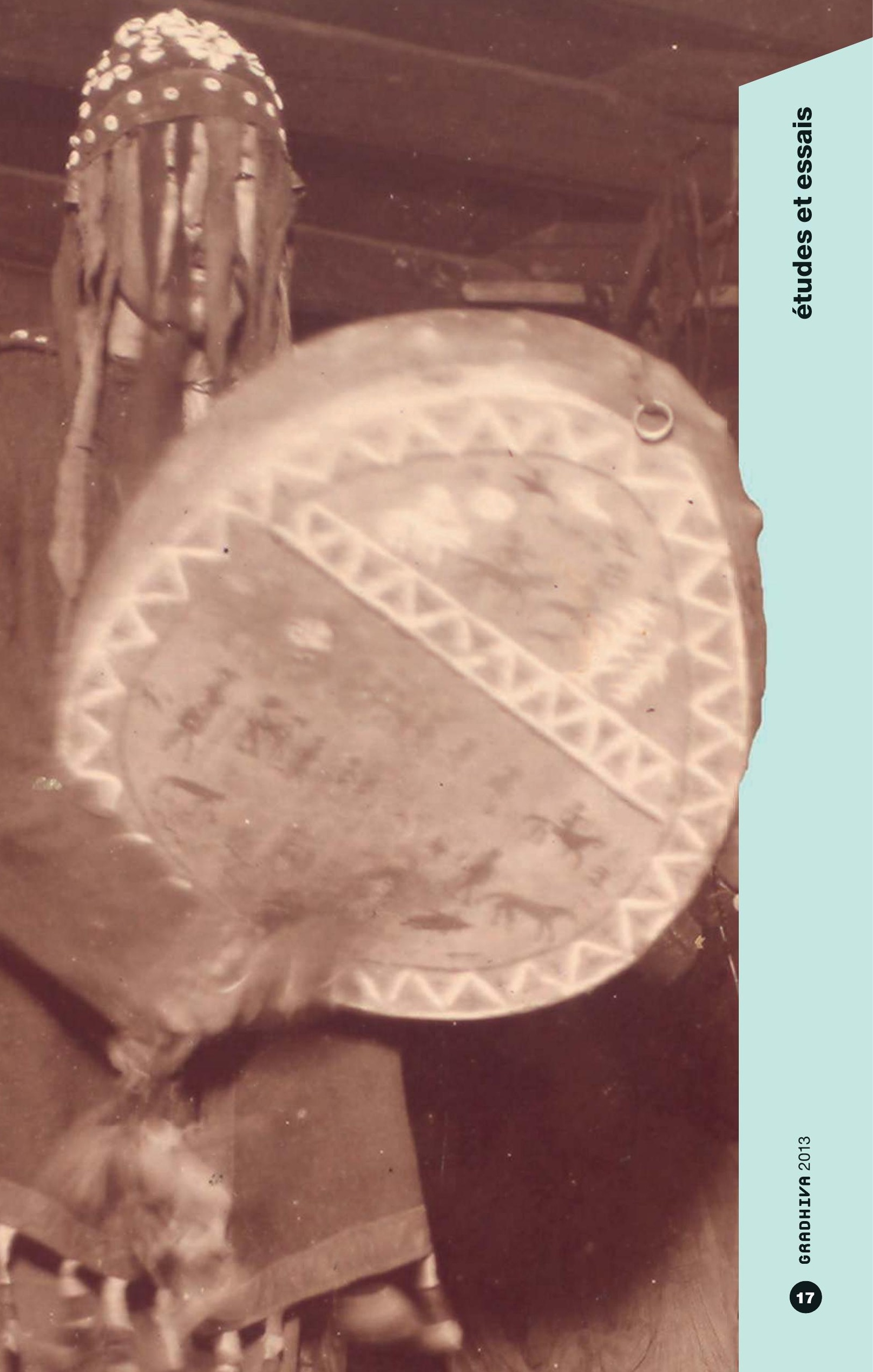




\section{Dessins chamaniques et espace virtuel dans le chamanisme khakasse}

par Charles Stépanoff

Les tambours rituels, attributs principaux des chamanes de Sibérie, sont souvent ornés de nombreuses figures peintes.

Les interprétations sémiotiques de ces dessins ont jusque-là laissé dans l'ombre certaines récurrences séculaires de composition particulièrement intrigantes. En s'appuyant sur une approche sensorimotrice, cet article défend l'hypothèse selon laquelle l'organisation des dessins modélise une coordination entre corps, espace réel et espace virtuel sur la scène rituelle. 
Les tambours utilisés pour accompagner les chants rituels sont les attributs caractéristiques des chamanes d'Asie septentrionale. Si divers soient-ils dans leur forme et leur taille, les tambours nord-asiatiques ont pour trait commun de présenter un côté ouvert et un côté fermé par une membrane. Chez certaines populations, la surface de la membrane s'orne d'une profusion de figures peintes de personnages, d'animaux et d'arbres.

Les études concernant ces images énigmatiques ont été généralement inspirées par une méthode qui peut être qualifiée de sémiotique et de cosmographique. Sergej Ivanov (Ivanov 1954 et 1955), Vilmos Diószegi (Diószegi 1998 [1978]; Lot-Falck et Diószegi 1973) et Éveline Lot-Falck (Lot-Falck 1961) ont cherché à identifier la signification originelle de chaque figure en l'intégrant à une «vision du monde » locale. Diószegi s'efforce ainsi de mettre au jour l'«arrière-plan idéologique» que les figures «reflètent» (Diószegi 1998 [1978]: 251). De la même manière, Vladimir Basilov considère que les dessins «reflètent les représentations sur le monde environnant et les esprits» (Basilov 1984: 87). Leonid Potapov estime de son côté que, «par le langage des dessins, sorte d'écriture pictographique, les postulats théologiques du chamanisme altaïen étaient reflétés sur la surface de la membrane du tambour » (Potapov 1991: 193).

Cette approche se heurte à plusieurs difficultés dès lors que l'on observe que les tambours ne donnent jamais du panthéon d'une population qu'un aperçu incomplet et biaisé. Variant souvent fortement d'un chamane à l'autre, ils ignorent les principales divinités et font figurer en revanche des figures mineures que les chamanes eux-mêmes sont quelquefois en peine d'identifier. La lecture cosmographique, qui réduit les dessins à une représentation iconique d'une représentation mentale du monde, présume transparents les rapports entre le niveau pictural et le niveau mental. Ce faisant, elle détache les dessins à la fois des instruments sur lesquels ils ont été peints et de leur contexte pratique d'usage pour les soumettre à une hypothétique idéologie collective. S'il est incontestable qu'ils ont des rapports avec certaines représentations mentales, la métaphore courante du «reflet» est loin d'offrir une explication satisfaisante de la nature de ces rapports.

Les remarques des chamanes eux-mêmes concernant le rôle des dessins sur leurs tambours n'ont pas suscité dans les études spécialisées l'attention qu'elles méritaient, probablement parce qu'elles s'accordent mal avec la fonction expressive qui leur est prêtée. Les chamanes ne paraissent pas considérer que les dessins délivrent un message, ils affirment qu'ils les aident plutôt à «s'orienter dans leur voyage», à «avancer» (chez les Khakasses, Potapov 1981: 134-135), à «s'orienter dans les pays obscurs» (chez les Évenks, Ivanov 1954: 177). Or qu'est-ce que «s'orienter», sinon établir une coordination cognitive et sensorielle particulière entre son propre corps et l'espace environnant? Les indications des utilisateurs des tambours suggèrent ainsi que les dessins pourraient s'éclairer à la lumière des relations entre corps et espace dans le contexte particulier de l'action rituelle.

L'enjeu est ici, pour reprendre les termes de Carlo Severi, de passer “d'une typologie des représentations à l'identification d'une logique des relations représentée par l'image au sein d'une tradition » (Severi 2011: 11). 
L'analyse menée par Severi (2007) des dessins utilisés dans les traditions chamaniques amérindiennes montre que, s'il s'agit bien de pictographies, leur rôle n'est pas pour autant de «représenter» de façon sémiotique une vision du monde. Ces dessins constituent plutôt un «art de la mémoire» dédié à la performance rituelle. Leur organisation spatiale est en effet en rapport étroit avec la structure séquentielle des chants rituels qu'ils aident à mémoriser. L'essentiel est alors «la relation qui s'établit entre une iconographie relativement stable et un usage rigoureusement structuré de la parole rituelle, entre iconographie organisée en forme paralléliste et mise en mémoire» (ibid. : 198).

Assurément, la compréhension des dessins sibériens ne progressera que si l'on s'interroge sur leur rapport avec le déroulement de l'action rituelle. Cependant, ces images ne se laissent pas déchiffrer à la façon d'une pictographie amérindienne: elles n'ont pas d'ordre de lecture et elles accompagnent de chants nombreux et divers, de sorte qu'elles sont de peu de secours pour la mémorisation d'une structure chantée. L'ordre de la parole ne semble pas être le point d'ancrage principal des images dans le rituel, ou du moins pas le seul.

Les gestes du chamane contribuent autant, voire plus, que ses chants à évoquer au cœur de la scène rituelle l'espace invisible qui sert de cadre mental à l'action. Le rituel chamanique sibérien a en effet ceci de particulier qu'il accomplit une coordination réglée entre un espace visible, généralement la maison du malade, et un espace virtuel inaccessible à l'assistance, où le chamane est supposé négocier et lutter en faveur de son client. Ceci va nous amener à proposer une approche «sensorimotrice» des tambours. C'est dans la motricité que corps et espace se coordonnent, et cette motricité s'accompagne toujours d'un engagement des sens (Berthoz 1997; Warnier 1999). Notre hypothèse est que les images des tambours jouent un rôle majeur dans la redéfinition du cadre spatial de l'action gestuelle du chamane. À ces images sont couplés des schémas sensorimoteurs conventionnels coordonnant proprioception, espace réel et espace virtuel.

Nous examinerons ici le cas des tambours khakasses, qui sont parmi les plus richement ornés et les mieux documentés de Sibérie. Les Khakasses, autrefois appelés Tatars de Minoussinsk, sont un peuple turcophone réunissant plusieurs groupes, les Kačin, les Beltir, les Kyzyl, les Sagaj et les Kojbal, établis dans la vallée du haut lenisseï sur les contreforts septentrionaux des monts Saïan. Ils ont été formellement christianisés à partir du XIX $x^{e}$ siècle, sans que l'Église parvienne à faire sensiblement reculer les pratiques chamaniques. La fabrication traditionnelle des tambours a cessé à l'époque des sanglantes répressions soviétiques dans les années 1930 (voir Stépanoff 2009).

\section{Les tambours khakasses}

Ivanov a recensé dans les collections des musées russes cinquante tambours khakasses portant des dessins lisibles. Au total, ce sont environ mille cinq cents figures qui se laissent reconnaître sur ces instruments (Ivanov 1955: 178). Des commentaires détaillés de chamanes expliquant les dessins ont été relevés de la fin $d u x x^{e}$ siècle au milieu du $x x^{e}$ siècle par 

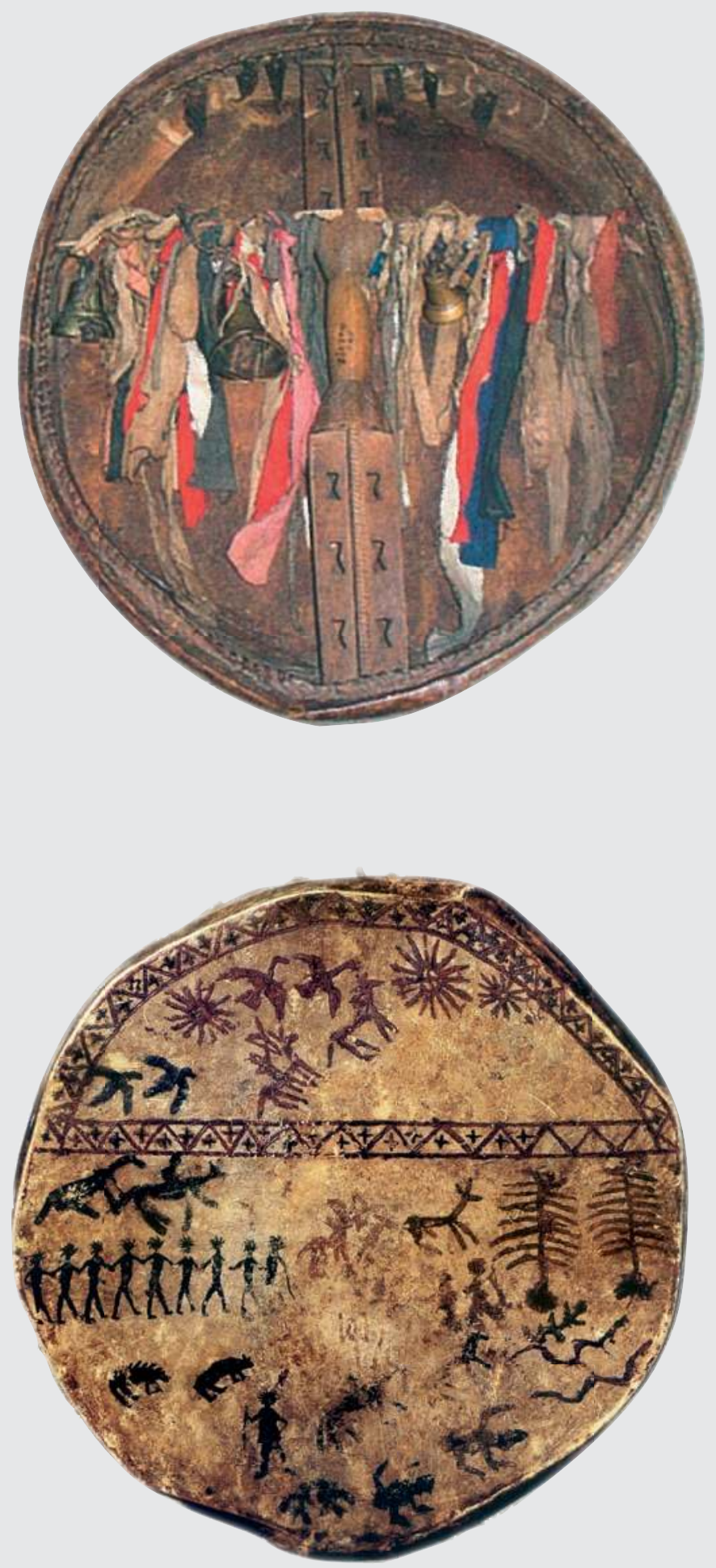
les ethnologues khakasses Nikolaj Katanov (Katanov 1897, 1907 et 2000), Stepan Majnagašev (archives du musée d'Anthropologie et d'Ethnographie [Kunstkamera], Saint-Pétersbourg) et Viktor Butanaev (Butanaev 2006), et par les Russes Dmitrij Klemenc (Klemenc 1890) et Potapov (Potapov 1981).

Le tambour (tüür) était l'instrument principal des chamanes khakasses. Une personne reconnue comme ayant les qualités de chamane entrait en fonction par le rituel d'animation de son tambour, spécialement fabriqué pour elle par son entourage. De forme ronde, les tambours khakasses ont un diamètre de $70 \mathrm{~cm}$ et plus. Le cadre cylindrique, en bois de saule, est traversé par un manche vertical de bouleau percé de part en part de trous triangulaires. C'est par ces trous que, à l'appel du chamane, les esprits sont censés pénétrer dans le tambour et ressortir de l'autre côté après la séance chamanique (Klemenc 1890: 25). Une tige métallique sur laquelle sont suspendus cloches, pendeloques et rubans traverse horizontalement le manche. La membrane est faite d'une peau d'animal: cheval hongre, cervidé ou bouquetin. Le battoir (orba) en bois de cerf maral est couvert de fourrure et orné de rubans.

La face externe de la membrane est couverte de figures peintes en noir, rouge et blanc. Dans la composition la plus commune, le cercle du tambour est traversé par une bande horizontale parcourue de zigzags triangulaires et placée un peu plus haut que le diamètre (fig. 2). Si l'on compare les faces interne et externe, on s'aperçoit que la bande se situe à peu près au niveau de la traverse métallique à l'intérieur du tambour (fig. 1). Cette bande constitue pour certains chamanes une image des couches superposées de la terre, les zigzags correspondant aux montagnes. Le grand secteur situé sous la bande est associé aux milieux terrestre, aquatique et souterrain, tandis que le petit secteur supérieur correspond au milieu céleste. Ce dernier est clos par un arc longeant le bord du tambour et parcouru lui aussi de zigzags: un arc-en-ciel d'après les chamanes. Les dessins remplissant ces secteurs puisent dans un répertoire de figures récurrentes, certaines d'entre elles occupant des positions très stables dans la composition alors que d'autres sont plus mobiles.

\section{Secteur supérieur}

Le sommet du secteur supérieur est occupé par des astres: le soleil, la lune, les «Trois Biches», c'est-à-dire la constellation d'Orion, l'Étoile du soir (Ir Solbany), I'Étoile du matin (Tan Solbany) et la Grande Ourse (Čedigen). Ces astres sont réputés se situer «sous» la demeure de Kudaj, le dieu céleste créateur, qui n'est pas représentée. Sous les astres se trouvent des oiseaux (deux aigles noirs, un coucou, parfois des oiseaux blancs) souvent placés du côté droit du tambour, dans les environs du sommet d'un ou deux arbres. Les chamanes khakasses expliquent que ces volatiles les aident à voyager vers le ciel et à soigner les maladies des yeux.

Le secteur supérieur compte plusieurs cavaliers: souvent un cavalier blanc sur un cheval blanc, se rendant chez Kudaj, et un cavalier rouge sur un cheval rouge armé d'un arc. Ce dernier représente l'esprit Seigneur Gaucher (Han Solagaj) ou un serviteur «se rendant» chez ce seigneur. D'après les chants que les chamanes lui adressent, on sait qu'il est le fils de l'empereur

\section{ci-contre}

fig. 1

Tambour khakasse, faces interne et externe. Saint-Pétersbourg, musée russe d'Ethnographie. 
de Chine et qu'il habite en pays touva. À proximité des cavaliers est dessiné un cervidé, un bouquetin ou un cheval, représentant l'animal dont la peau a été utilisée pour faire la membrane du tambour. Ce dessin est très important car il indique que l'âme (čula) de l'animal est présente dans le tambour et que celui-ci est donc vivant (Ivanov 1955: 202; Butanaev 2006: 97).

\section{Secteur inférieur}

Dans le secteur inférieur du tambour se rencontrent différentes figures noires de cavaliers, chevaux et piétons, globalement considérés comme des serviteurs ou des envoyés du seigneur Erlik, le maître du monde inférieur où demeurent les défunts (Katanov 2000: 371). Parmi eux on identifie Tuma le Noir (Kara Tuma), un cavalier protecteur des chevaux moreaux. Dans les invocations qui lui sont adressées, il est décrit comme un Mongol noir (Kara Mool) venu de Mongolie en traversant le pays chor, avec un serpent noir comme cravache. Ce «Mongol» est chargé de la protection du bétail contre les maladies (Butanaev 2006: 60; Ivanov 1955: 204).

Tout en bas, plutôt à droite et au centre, dans les environs des racines des arbres, on distingue des ours, des grenouilles, des serpents, des lézards et des poissons, tous en noir. Batraciens et reptiles sont soumis au «maître jaune des moutons» (kojdyn saryg yzygy) [Katanov 1907: I, 566]. Ils peuvent en outre mener le chamane chez Erlik. Ils sont sollicités notamment pour soigner les maladies des jambes ou les pathologies féminines. Les brochets guérissent les maladies abdominales et l'hydropisie.

Certaines figures importantes paraissent pouvoir se situer indifféremment dans le secteur supérieur ou inférieur. C'est le cas de deux bouleaux, placés en haut ou en bas, mais presque toujours dans la partie droite du tambour. D'après les chamanes, ils les aident à «monter vers le ciel».

On rencontre souvent sur les tambours khakasses une série de personnages se tenant par la main. Ce sont les «sept filles jaunes de la montagne", parfois accompagnées de "neuf garçons noirs». Ces enfants d'esprits maîtres des montagnes ont un rôle d'intermédiaires dans les négociations du chamane avec leur père. Enfin, comme ailleurs dans l'AltaïSaïan, le chamane est lui-même souvent représenté parmi les dessins, armé d'un arc ou de son tambour.

De cette première vue d'ensemble ressort l'absence notable des entités dominantes du "panthéon" khakasse, principaux dédicataires des rituels: les maîtres des montagnes, le dieu céleste Kudaj, le dieu infernal Erlik. Les êtres représentés sont plutôt des serviteurs, des guides et des intermédiaires maîtrisant des routes dont les destinations ne sont pas figurées. Ainsi l'espace peint n'est pas tant à regarder comme un cosmogramme, une "carte du monde" définissant des territoires délimités, que comme un champ vectoriel traversé d'itinéraires et d'orientations contrastives.

ci-contre

fig. 2

Tambour khakasse (sagaï).

Musée de Minoussinsk

(Ivanov 1955: 197, fig. 14).

\section{Oppositions récurrentes}

La disposition générale que nous avons décrite se retrouve sous une forme plus élémentaire dans un relevé de tambour baraba du xvIII siècle (fig. 3). En raison de sa simplicité et de son ancienneté, ce tambour peut 


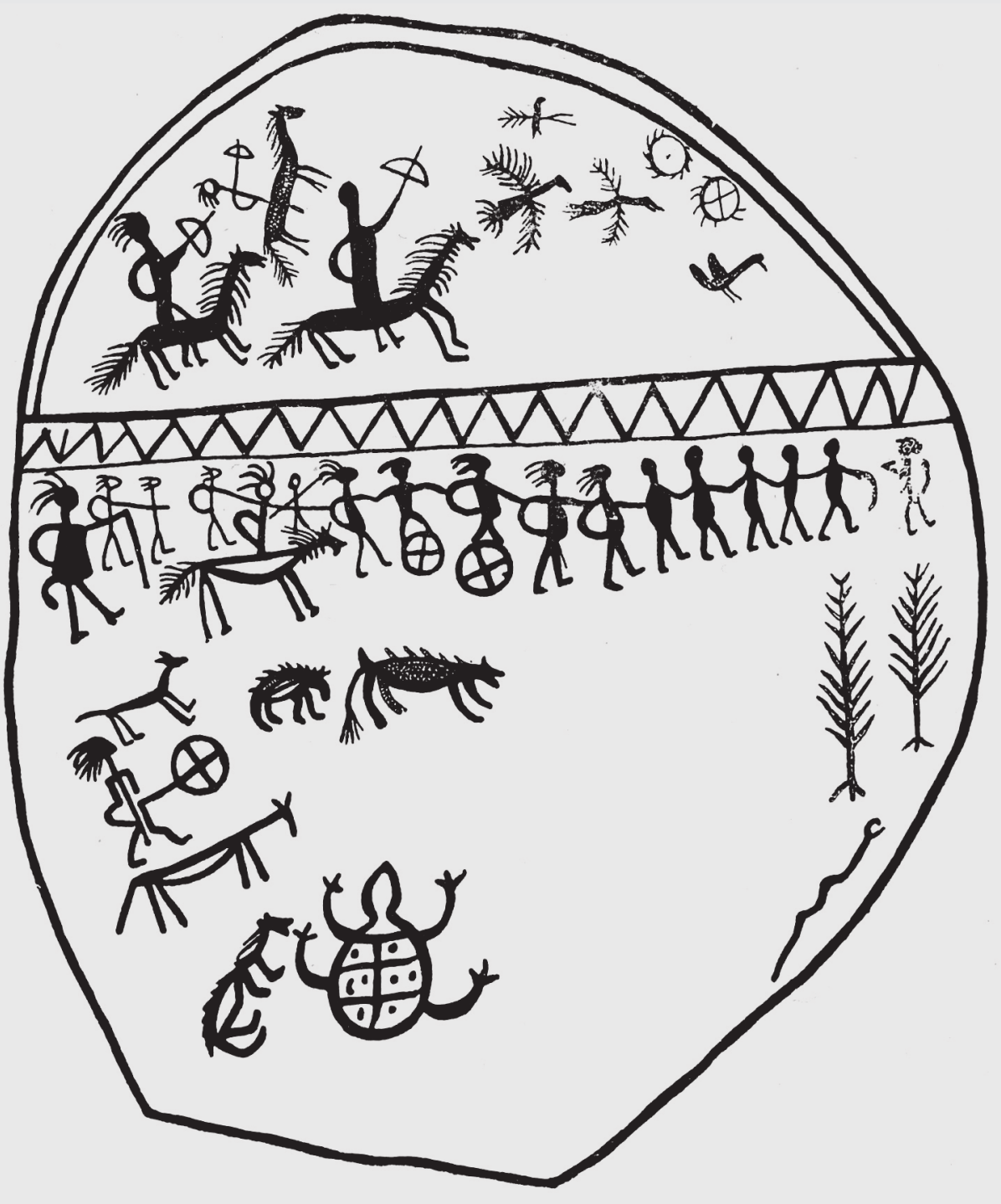


1. Chez les Altaïens, il est dit explicitement que le bouleau dessiné est celui dont on a fait le manche du tambour (Anohin 1924: 56).

\section{ci-contre}

fig. 3

Tambour des Turcs baraba (Sibérie Occidentale). Manuscrit de Daniel Gottlieb Messerschmidt, XVIII ${ }^{\ominus}$ siècle (Ivanov 1979: 140, fig. 151).

fig. 4

Freda Heyden, Tambour khakasse, début du xx siècle, aquarelle. Saint-Pétersbourg, musée d'Anthropologie et d'Ethnographie (Oppitz 2007: 76). être regardé comme une Urform dont sont dérivées non seulement les compositions khakasses modernes, mais aussi celles de peuples turcs tatars apparentés comme les Téléoutes et les Chors (voir Lot-Falck et Diószegi 1973).

Ces tambours que l'on peut qualifier de type «tatare" sont traversés de lignes de tension d'une grande constance sur les plans vertical et horizontal. Entre le haut et le bas s'opposent le céleste et le terrestre, le sec et l'humide, le clair et l'obscur. L'indication par les chamanes khakasses des maladies dont sont spécialistes certaines des figures représentées y ajoute une correspondance avec le corps humain: les oiseaux du secteur supérieur sont liés à la tête, alors que les animaux de la partie basse sont spécialisés dans le ventre et les jambes.

D'après le chamane kačin Roman, au cours du rituel d'animation du tambour le nouveau chamane entend l'esprit de la montagne Kara-tag lui dire ceci: «Tu soigneras les gens; soigne les maladies pures avec le protecteur des chevaux et les maladies impures avec le protecteur des moutons, des lézards et d'autres auxiliaires. " (Potapov 1981: 133) Les maladies "pures", concernant la partie haute du corps, sont donc à la charge des esprits maîtres des chevaux, ces cavaliers représentés dans les parties médiane et supérieure du tambour, tandis que les maladies impures situées dans le bas du corps, plus particulièrement les maladies gynécologiques, relèvent du maître des moutons, qu'on a vu associé aux batraciens et aux reptiles du bas du tambour. II existe donc une correspondance entre la verticalité du tambour et celle du corps humain établie par l'intermédiaire de l'ordre spatial du paysage et de ses habitants représentés sur la membrane.

Alors que l'organisation verticale des tambours est bien connue, la possibilité d'un ordre perpendiculaire des figures, selon l'axe horizontal, n'a guère été envisagée. Ivanov a noté que les arbres se situent généralement dans la partie droite du tambour, sans toutefois pouvoir expliquer cette régularité (Ivanov 1955: 215). Ils se trouvent en fait en opposition diamétrale avec les cavaliers qui occupent le bord gauche et s'étendent vers le centre. Cette opposition, d'une force et d'une stabilité frappantes, se distingue très nettement dans le tambour baraba du xvIII ${ }^{e}$ siècle (fig. 3), mais aussi dans les tambours modernes des Téléoutes et des Altaïens du Sud. Aucun commentaire de chamane ne fournit d'explication sur cette combinaison spatiale typique des tambours de la famille tatare.

Les choses s'éclairent si l'on s'intéresse au rapport de ces figures non plus seulement avec la cosmologie, mais avec les gestes concrets des chamanes au cours du rituel. Dans sa main gauche, le chamane tient le manche du tambour qui est fait en bois de bouleau, précisément l'arbre représenté sur le bord droit ${ }^{1}$. De sa main droite, il agite son battoir, qualifié de "cravache" (hymčy). Dans les techniques de monte turco-mongoles, c'est effectivement de la main droite qu'est tenue la cravache. Le côté droit du chamane est donc associé au mouvement et à la monte, alors que le côté gauche est lié à la stabilité et au bois de bouleau. Cette répartition des tâches des mains est universelle dans le chamanisme turco-mongol de Sibérie, ce qui explique certainement le positionnement stable des cavaliers et des arbres sur ces tambours. 

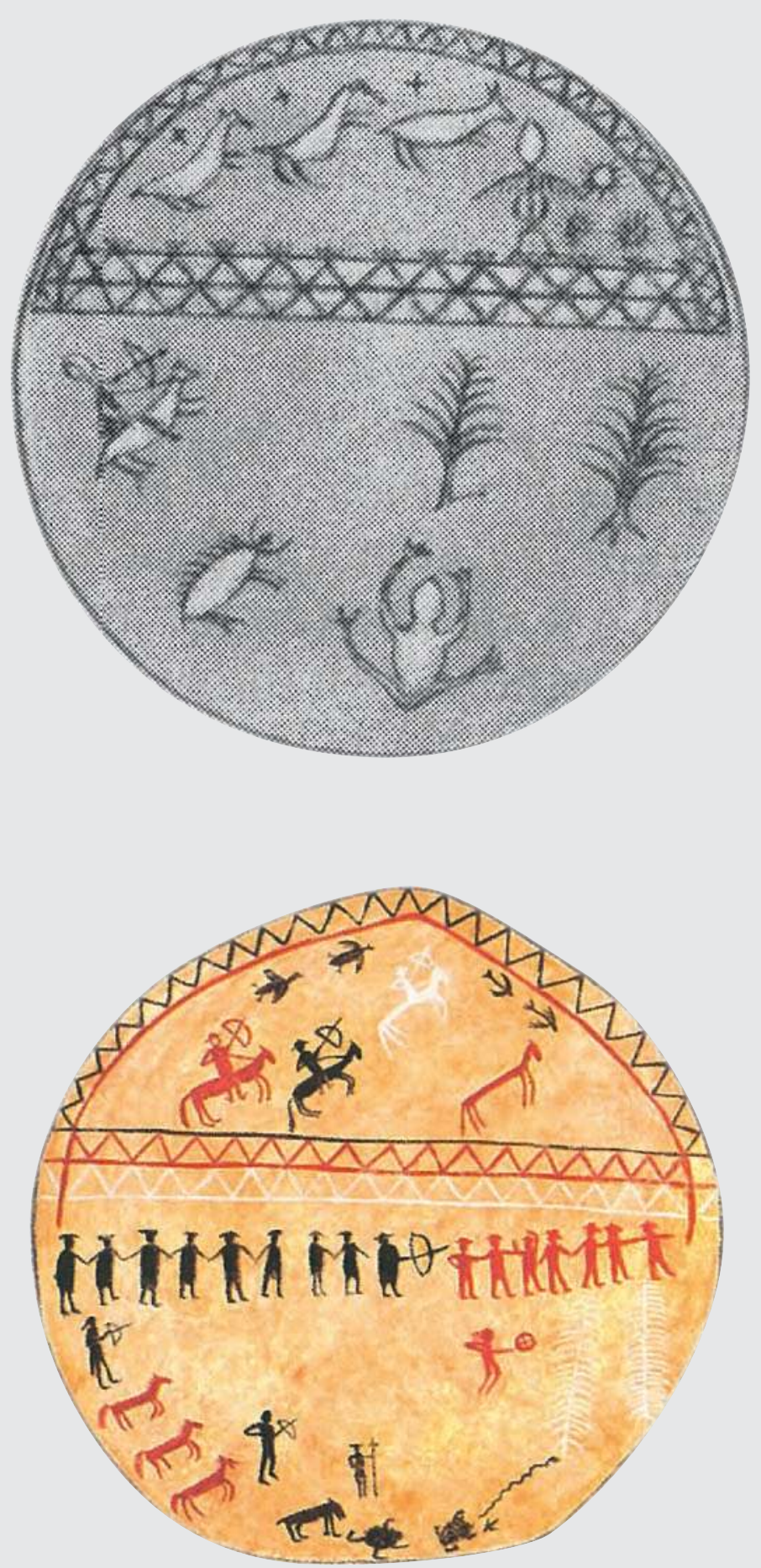
Au début de leurs rituels, les chamanes de l'Altaï-Saïan restent un moment face au feu, la tête dans le cadre du tambour, et battent la membrane de coups légers (fig. 5). À mesure que les esprits invoqués sont supposés se présenter, le chamane frappe et chante plus fort, jusqu'à se lever, marquant ainsi le début de son «voyage ( (čörerge) avec ses auxiliaires vers les montagnes voisines, le ciel ou l'enfer selon le cas. Concrètement, lorsqu'il a la tête dans le tambour, le chamane voit apparaître à contre-jour les dessins éclairés par le feu. Bien nettoyée, la peau de la membrane est en effet parfaitement translucide. Les yeux cachés derrière la membrane, le chamane se coupe de l'interaction avec le public pour entrer ostensiblement dans un champ relationnel nouveau. II laisse les dessins et le battement de la membrane qui résonne près de ses oreilles envelopper ses perceptions visuelles et auditives.

Pour l'officiant comme pour l'assistance, la partie droite de la membrane peinte s'associe alors clairement à son bras gauche et la partie gauche à son bras droit. Ce parallélisme entre latéralité du tambour et latéralité du corps du chamane est scellé par le positionnement des arbres et des cavaliers dans le dessin.

II existe une autre régularité frappante dans l'axe horizontal: sur presque tous les tambours, les figures dessinées regardent vers la droite du cadre. À nouveau, on ne peut expliquer cette récurrence que si l'on prend en considération les gestes et postures du chamane. Lorsque le chamane s'est élancé dans son «voyage», il lui arrive de placer son instrument entre ses jambes et de le chevaucher (fig. 6). En effet, dans les traditions turco-mongoles, le tambour (et à travers lui l'animal qui a donné sa peau pour faire la membrane) est souvent désigné et utilisé comme une monture du chamane. Quand un chevauchement est mimé, l'instrument, tenu de la main gauche, se retrouve placé la membrane contre la jambe droite de l'officiant. La partie droite du dessin se positionne ainsi à l'avant du corps de celui-ci et la partie gauche à l'arrière. Tout porte à croire que si les personnages sont tournés vers la droite, et au premier chef la figure représentant l'animal dont la peau a été utilisée, c'est de façon à avancer dans la même direction que le chamane. Cette hypothèse est confirmée par un détail très révélateur d'un tambour khakasse conservé à la Kunstkamera de Saint-Pétersbourg: sur la membrane en peau de cerf maral les poils ont été en partie maintenus, or leur orientation permet de voir que l'avant de l'animal se situe du côté droit et l'arrière du côté gauche. L'inventaire contient une information recueillie auprès du chamane propriétaire de l'objet: «Le chamane monte ce cerf maral, c'est pourquoi la partie avant de la peau est orientée dans le sens où vont toutes les figures. " (musée d'Anthropologie et d'Ethnologie, inventaire 2164-1; voir Ivanov 1955: 180) Cela signifie que l'avant de l'animal se situe du côté droit du tambour, de sorte que la monture regarde dans la même direction que le chamane lorsque celui-ci le chevauche. Et c'est pour cette même raison que les figures peintes s'avancent vers la droite.

La disposition des figures sur l'axe gauche-droite est donc structurée par deux ensembles d'évocations. D'une part, l'ordre des figures est en rapport de projection avec la latéralité fonctionnelle du corps du chamane. 
Chez les voisins altaïens des Khakasses, l'association du tambour avec le corps chamanique est particulièrement explicite puisqu'une figure anthropomorphe représentant un chamane ancêtre organise l'ensemble de la composition. La superposition du corps chamanique et du tambour apparaît aussi parfaitement sur le pétroglyphe d'Oglahty, en région khakasse (fig. 7). Au cours des rituels, cette projection est donnée à voir lorsque le tambour est parallèle à la ligne des épaules du chamane, c'est-à-dire lorsque celui-ci frappe doucement. Mais l'orientation individuelle des figures vers la droite renvoie par ailleurs à une autre position classique, lorsque le tambour fait office de monture enjambée par le chamane et, plus simplement, quand l'officiant bat fort et vite du battoir et que l'instrument se trouve perpendiculaire à l'axe des épaules. Ce dernier n'est alors plus un double, mais un cheval compagnon. Identification parallèle et complémentarité perpendiculaire ne sont pas en contradiction: elles correspondent aux deux positions extrêmes du tambour entre lesquelles le chamane va et vient au cours du rituel dans une alternance de pauses et d'excitations.

\section{Les esprits dans la yourte}

Nous avons discerné quelques coordinations entre tambour et corps. Certaines remarques des chamanes khakasses suggèrent qu'il existe en outre des correspondances entre l'ordre spatial des dessins et celui de la yourte où sont menés les rituels. Le chamane beltir Petrov expliquait que tout en bas de son tambour figure un personnage, le «maître de l'eau », que les chefs de famille khakasses honorent en posant un plat de viande d'agneau chaude près de la porte, "pour que cela fume vers le maître de l'eau». Dans la yourte, il se trouve en effet que l'on conserve l'eau du côté droit de la porte en entrant. Petrov indiquait aussi que les sept étoiles figurant dans le ciel du tambour sont évoquées par les chamanes dans le chant qu'ils adressent à kök yzyk, une amulette installée à distance de la porte, dans la partie sud ou sud-ouest (Katanov 1907: I, 565). Enfin, deux chamanes kačin, Apčaj et Roman, interrogés à cinquante ans d'écart, désignent l'ours figurant dans le bas de leur tambour comme le «gardien de l'entrée de la yourte » (ibid.: I, 598; Potapov 1981: 135).

Ces associations ponctuelles sont-elles fortuites ou indiquent-elles une correspondance générale entre dessins et yourte? Si cette dernière hypothèse est correcte, le tambour s'avérera coordonné à la fois au corps chamanique et à l'espace environnant, ce qui pourra éclairer sa fonction d'instrument d'«orientation » affirmée par les chamanes.

La yourte et le tambour sont deux cercles orientés, horizontal pour la première, vertical pour le second. Chez les peuples nomades d'Asie septentrionale, le changement régulier de site concret d'habitat est compensé par des principes abstraits extrêmement stables d'orientation et d'organisation de l'espace domestique. Lorsque au cours du XIX siècle les Khakasses ont abandonné les yourtes de feutre pour des structures de bois polygonales, ils ont conservé l'ordonnancement spatial ancien.

La terminologie distingue dans la yourte quatre grandes zones (fig. 8). Le coin de la porte est orienté à la façon turque, vers l'est, appelé isker, «devant». Au centre de la yourte est installé le foyer surmonté d'un trou 


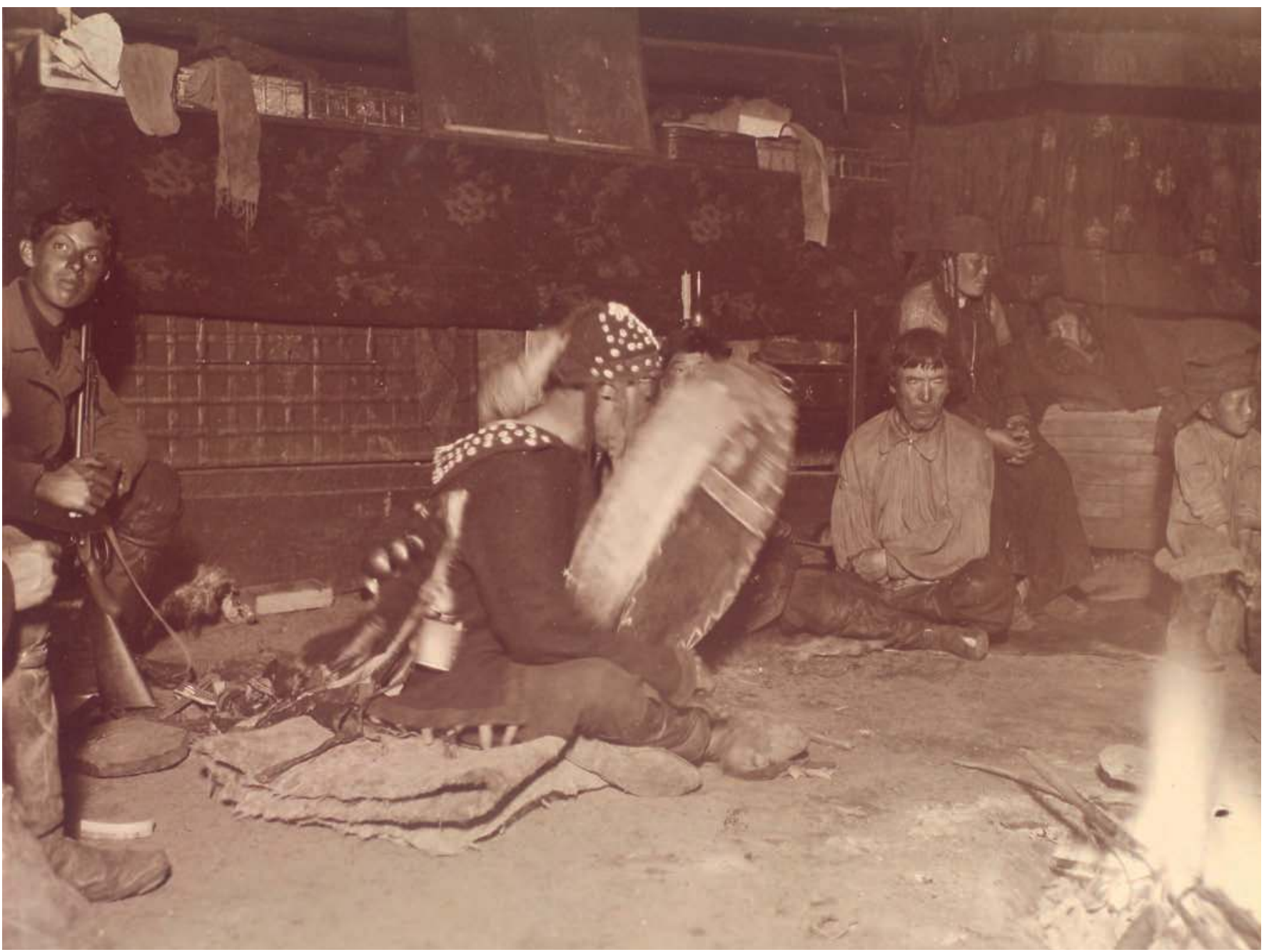

fig. 5

Ø.M. Olsen, Chamane

khakasse face au feu, 1914.

Bibliothèque nationale de

Norvège. 


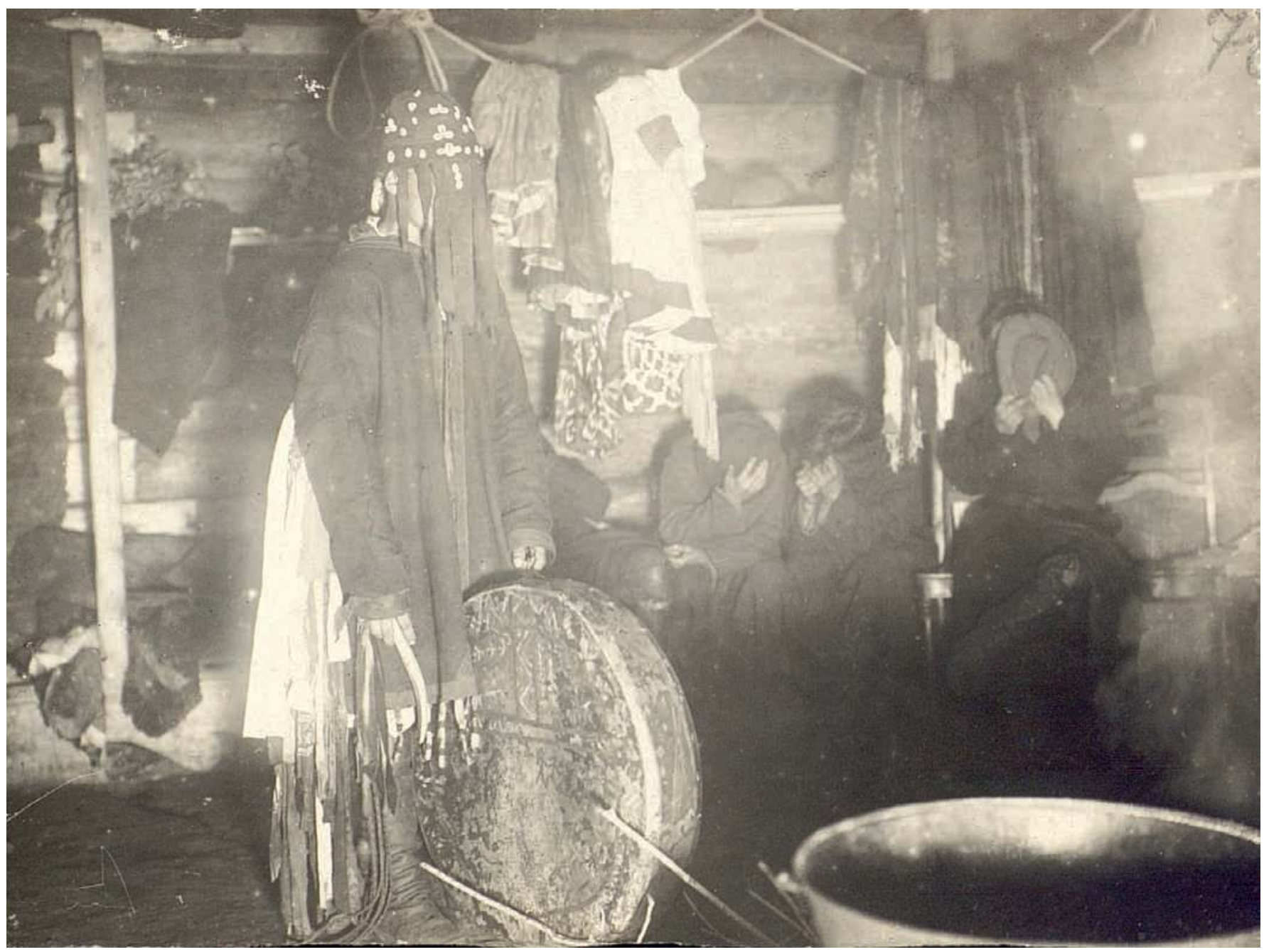

fig. 6

S.D. Maynagashev, Chamane en transe chevauchant son

tambour: adoration de la divinité du feu, début du $x x^{\mathrm{e}}$ siècle. Saint-Pétersbourg, musée d'Anthropologie et d'Ethnographie

(Kunstkamera), Académie des sciences de Russie, collection Pierre le Grand, coll. N ${ }^{\circ} 2410-78$. 
2. Viktor Butanaev, communication personnelle, 30 septembre 2011.

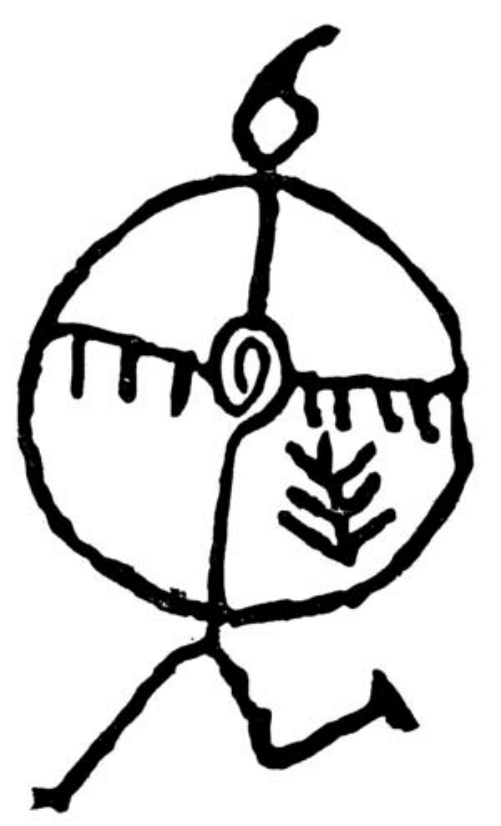

fig. 7

Pétroglyphe d'Oglahty, Khakassie, $x V I I^{e}-{ }^{-X I} x^{e}$ siècle (Kyzlasov et Leont'ev 1980: 144). On reconnaît le bonnet typique des chamanes khakasses.

La traverse métallique avec ses pendeloques fusionne avec les bras du personnage. Comme d'habitude, l'arbre figure du côté du bras gauche du chamane. à fumée dans le toit. Au-delà du feu, à l'opposé de la porte, s'étend le coin d'honneur, tör, avec le lit des maîtres. À ses pieds s'assoient les anciens et les hôtes d'honneur, idéalement la face éclairée par le soleil levant. C'est le point de vue de ces personnes qui détermine les valeurs associées à l'espace intérieur. À leur droite s'étend la partie pure et masculine, appelée en khakasse üstünzaryh, "sud", littéralement «le côté haut» (üstü sary). À gauche, la partie opposée, féminine et impure, est nommée altynzaryh, "nord», littéralement "le côté bas» (alty sary). Sur les murs méridionaux sont fixés les instruments masculins: le fusil au sud-ouest et le harnachement des chevaux au sud-est, près de la porte. Le long des murs septentrionaux s'étendent les instruments des femmes, étagères de vaisselle et ustensiles de cuisine (Katanov 1897: 23, note 1). Si le sud est "haut» et le nord "bas", c'est certainement du fait des associations liées dans le monde turc à ces orients, mais aussi en raison de la géographie du pays khakasse, drainé par le lenisseï qui descend des steppes touvas au sud vers la taïga septentrionale.

En fait, une opposition verticale se retrouve aussi de façon latente entre la porte et le coin d'honneur (tör). Le tör est nommé «tête du feu» (ot pazy) alors que le coin de la porte est «l'arrière du feu " (ot soo ${ }^{2}$ ). Chez les Altaïens, cette opposition s'exprime par les termes «tête du feu» (ottyn bažy) et «jambes du feu» (ottyn budy). On invite à s'asseoir dans le tör un hôte de marque qui se tient près de la porte en lui disant: "Asseyez-vous plus haut!» (Örü oturar) [Tadina 2006]. Cette terminologie altaïenne est en cohérence avec la disposition du corps lorsque l'on s'allonge dans une yourte: on s'efforce d'avoir la tête vers le coin d'honneur et les pieds vers la porte.

Par conséquent, l'opposition de valeur la plus forte se situe entre le quart nord-est, secteur deux fois inférieur car féminin et cadet, et le quart sud-ouest, deux fois supérieur car masculin et aîné. C'est précisément dans le secteur sud-ouest qu'est installé l'autel domestique, appelé kudaj pulii, «la place de Dieu-Ciel». C'est là que sont suspendus les icônes orthodoxes et le tambour chamanique lorsqu'un chamane est en visite. À l'opposé, dans le coin nord-est, sont rangés des seaux contenant les réserves d'eau et les produits laitiers. Nous retrouvons ainsi dans la yourte, comme pour le tambour, une opposition entre le haut céleste et le bas aquatique.

L'ordre spatial de la yourte s'enrichit d'évocations complexes par la présence sur ses murs d'amulettes représentant des esprits protecteurs des humains et du bétail. Chaque amulette est spécialisée dans un soin ou une protection particulière et reçoit une nourriture spécifique. En outre, à chacune est réservé un emplacement plus ou moins précis (Adrianov 1909; Butanaev 2003; Katanov 1907).

Les murs méridionaux sont ornés d'esprits cavaliers déjà rencontrés sur le tambour, tandis que sur les murs septentrionaux trouvent leur place des esprits liés aux femmes. Certains de ces derniers sont évités par les hommes qui les qualifient de «mauvais esprits»: ainsi la "femme esprit téléoute» (tileg-tös), responsable des maladies des pis de vache et des maux de ventre, et l'ours situé au nord-est (Jakovlev 1900: 107; Katanov 1907: I, 425). 
Plusieurs de ces amulettes n'ont pas leur pendant sur le tambour, ainsi la mère Ymaj, protectrice des femmes et des enfants. Située au nord-ouest, dans la partie féminine du coin d'honneur, elle n'a de rapport qu'indirect avec un élément constant des tambours khakasses, les bouleaux plantés dans la partie droite. À propos de ces arbres, un chamane expliquait:«Quand nous sommes nés de notre père Ülgen [dieu créateur, équivalent de Kudaj], ces deux bouleaux ont été envoyés avec la mère Ymaj. » (Katanov 1907: I, 565)

\section{Tambour et yourte}

Il existe une correspondance entre la position de certaines amulettes dans la yourte et l'origine géographique de l'esprit qu'elles représentent. Ainsi les cavaliers Tuma le Noir et Seigneur Gaucher se trouvent-ils dans la moitié méridionale de la yourte, précisément du côté d'où ils sont supposés venir, les steppes mongoles et touvas. Face à eux, côté nord, se tiennent la «femme esprit téléoute», dite aussi «esprit du nord» (altynzary tös), et l'«esprit toungouse» (tonaza tös), responsable des vents du nord. Or les Téléoutes sont établis au nord-ouest du pays khakasse et les Toungouses peuplent toute la taïga septentrionale. C'est aussi côté nord que sont installés d'autres représentants du monde de la taïga: l'ours, le renard et le putois. Bref, c'est toute la géographie environnant le pays des Khakasses qui se trouve exposée dans leur maison. La yourte n'est pas un espace hermétiquement fermé: avec ses amulettes commandant des routes, elle contient de nombreux points de départ vers l'extérieur.

En outre, on constate que les esprits du nord-est sont responsables de maladies liées aux parties basses du corps, alors que ceux du sudouest sont compétents pour le haut du corps. À partir de l'orientation de la yourte vers le levant, le réseau des amulettes crée un ensemble de correspondances implicites entre le plan de l'habitat, le corps humain, le paysage environnant et une géographie lointaine.

Dans toutes les régions khakasses, on retrouve sur l'axe sud-ouest/ nord-est une opposition entre deux éléments: l'autel du céleste Kudaj au sudouest et aba-tös, l'esprit ours, au nord-est. Ce dernier est en effet installé sur le côté féminin (nord) de la porte. Hibernant dans une tanière, l'ours est souvent associé dans le chamanisme sibérien au monde inférieur, à l'obscurité et à la féminité. Dans la yourte khakasse, il est figuré par un bâton couvert de peau d'ours, et orné d'un anneau de bronze évoquant probablement l'entrée de sa tanière et les lieux de communication entre les mondes qu'il surveille, porte et orifices inférieurs du corps. Nourri par une vieille femme, il est invoqué pour lutter contre la diarrhée et les maladies vénériennes.

Voisin de l'ours dans le bas du tambour, le serpent, chargé des douleurs aux jambes, et le cavalier Tuma le Noir se retrouvent dans la yourte près de la porte. Tuma le Noir y figure sous la forme d'une amulette composée de sept plumes noires de tétras lyre fixée près des portes côté sud, donc précisément au sud-est (ibid.: I, 611). Les "trois hommes noirs" figurés dans le secteur inférieur d'un tambour sont désignés comme des «intermédiaires» de la femme esprit téléoute installée dans le nord de 
la yourte (ibid.: I, 598). Ainsi, les figures situées dans le secteur inférieur du tambour se retrouvent dans l'est, le nord-est et le nord de la yourte.

L'équivalent de l'autel de Kudaj est à chercher sur le tambour dans la zone la plus haute, avec ses astres situés "sous la maison de Kudaj». Dessinés sous les astres, les oiseaux (hus tös) se retrouvent dans la région sud de la yourte avec pour fonction de soigner la tête, les yeux, les oreilles et les dents. Les cavaliers non noirs, comme Seigneur Gaucher le rouge, qui figurent dans le secteur supérieur du tambour se retrouvent aussi dans le sud et l'ouest de la yourte. La cavalière blanche Salyg, visible sur le tambour de la fig. 4 tout en haut du cercle, a sa place dans le secteur ouest de la yourte, entre le lit et l'autel (Adrianov 1909: 524; Ivanov 1955: 204). Le secteur supérieur de l'instrument correspond ainsi aux régions ouest, sud-ouest et sud de la yourte. D'une façon générale, l'axe haut-bas du tambour trouve son équivalent dans l'axe sud-ouest/nord-est de la yourte avec les oppositions communes: céleste/souterrain, sec/humide, clair/obscur.

Ceci n'est pas surprenant si l'on se souvient que, en dehors du rituel, le tambour est suspendu dans la partie sud-ouest de l'habitat. Ses dessins représentent en quelque sorte la disposition des esprits dans la yourte de son point de vue sud-ouest.

Qu'en est-il de l'axe gauche-droite du tambour, organisé autour de l'opposition cavalier-bouleau? Le monde des cavaliers des steppes concentré sur la gauche du tambour est clairement et exclusivement situé dans la moitié méridionale de la yourte. Par ailleurs, on se souvient de l'association entre les bouleaux du tambour et la déesse Ymaj, représentée dans la partie nord-ouest de la yourte. En outre, du côté nord sont concentrées des références animales et humaines au monde forestier: le renard, le putois, mais aussi l'esprit toungouse. Le contraste cavalier-bouleau du tambour se trouve dans la yourte converti en un contraste sud-nord entre steppe et taïga, animaux domestiques et animaux sauvages. La gauche du tambour se projette donc globalement dans le sud de la yourte et la droite dans le nord.

\section{Le rituel et ses espaces de référence}

Si yourte et tambour présentent tant de correspondances, c'est que ces surfaces obéissent à des schémas spatiaux communs les coordonnant à un paysage visible et invisible. Pour cette raison, le tambour peut apparaître comme une sorte de plan vertical de la yourte. Sur l'instrument de la fig. 4 , on voit un chamane tenant un tambour accompagné à sa gauche de sept personnages rouges, qualifiés de «filles jaunes", et à sa droite de neuf personnages noirs, appelés "garçons noirs". Il était fréquent au cours des rituels que les chamanes khakasses demandent à des garçons et des filles de les entourer. Au xvIII siècle, Johann Georg Gmelin observa une chamane khakasse (kačin)exécuter des danses avec sept hommes et sept femmes (Gmelin 1751-1752: III, 336-337). Dans un rituel publié par Butanaev (2006: 195), une chamane place neuf garçons du côté sud (masculin) de la yourte et sept filles du côté nord (féminin). Dans son chant, elle les décrit comme les "filles jaunes" et les "garçons noirs", enfants du maître de la montagne. Le tambour de la fig. 4 constitue ainsi une véritable modélisation réaliste de cette scène. 
OUEST

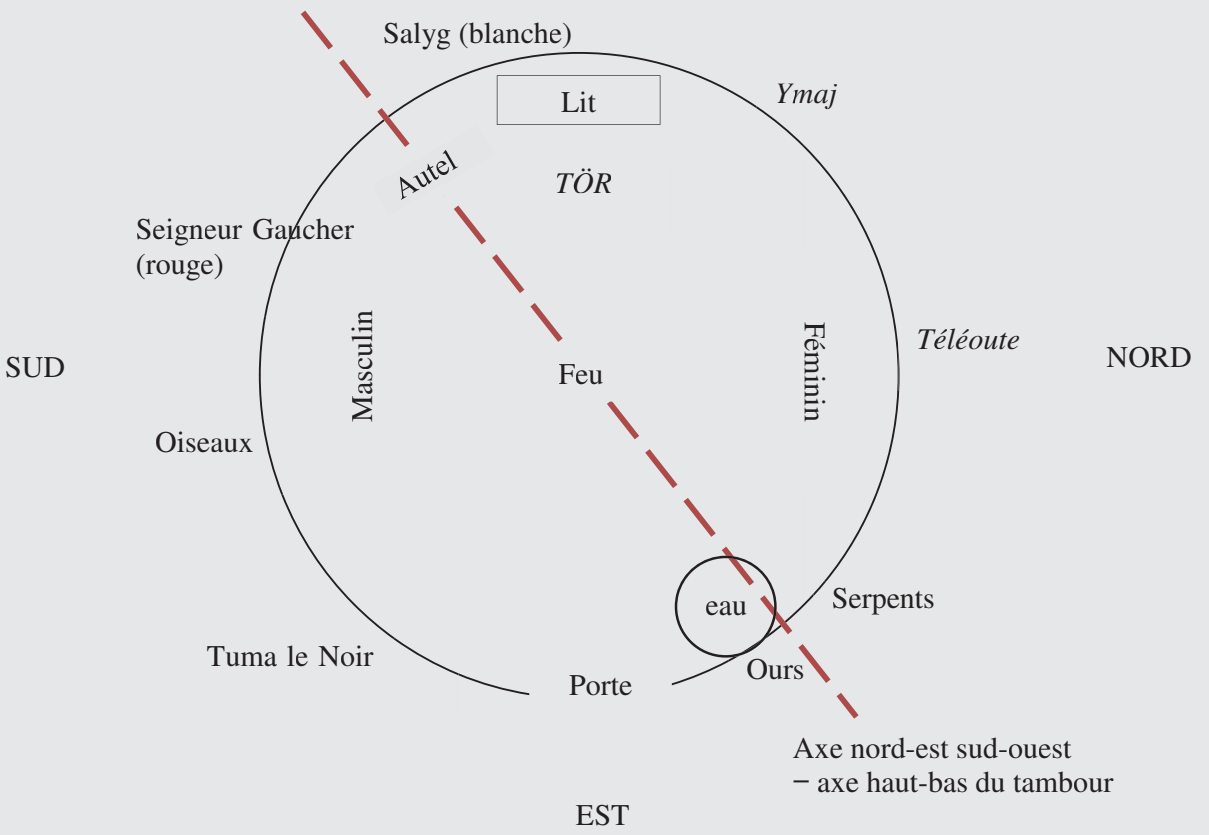

Afin d'éclaircir les rapports entre ces modèles spatiaux et l'action chamanique, nous allons examiner de plus près le rituel qu'accomplissait le chamane Pituk au début du xx siècle (ibid.: 196-207), en identifiant les espaces de référence auquels peuvent se rapporter les entités invoquées. Pituk se situe dans la yourte d'un malade environné de sa famille. La procédure de cure consiste à récupérer l'âme (hut) du patient supposée égarée et à renvoyer un esprit pathogène (ajna) installé dans son corps. Se tenant près de la porte, le chamane Pituk commence par appeler ainsi ses esprits: fig. 8

Emplacement des esprits dans la yourte. L'axe est-ouest correspondant à l'axe hautbas du tambour permet de retrouver le positionnement de la plupart de ces esprits sur l'instrument. En italique, esprits présents dans la yourte mais absents du tambour.

\section{Esprits de mon père-khan, \\ Envoyés de ma mère-khan, \\ Mes gens qui avez secoué mes épaules et mon cou, \\ Vous me faites sauter à fendre mes semelles, \\ Vous me faites hurler à me déchirer la voix. \\ Mes odžan [esprits] tenant le battoir, \\ Enroulez-vous sur ma main droite. \\ Mes tüben [esprits] tenant le tambour, \\ Entourez ma main gauche.}

Dans ce passage introductif classique, le chamane nomme de façon générique ses esprits en rappelant qu'il les a hérités de ses ancêtres et en remémorant les souffrances qu'ils lui ont imposées lors de la crise précédant son investiture. Le chant se poursuit par une description du costume rituel: 
Les bandelettes syzym de mon costume

Se sont tordues comme des roseaux;

Cinquante clochettes de mon costume blindé,

Vous chantez comme des oiseaux.

Puis commence l'évocation précise d'esprits individuels:

Mes serpents comme des flèches sifflantes,

Comme des flèches, sont tirés.

Mes grenouilles rugueuses,

Volant dans la clarté jaune,

Mes grenouilles aux doigts écartés,

Mes ours aux pattes tordues.

Les animaux cités dans ce passage, serpents, grenouilles et ours, se localisent tous dans la strate la plus basse de l'écosystème. Ils sont aussi associés à des espaces de référence immédiatement présents dans la scène rituelle. Ainsi, les serpents sont figurés dans le bas du costume khakasse sous la forme de bandes de tissu. Mais serpents et ours sont aussi présents dans la yourte près de la porte, où se trouve précisément le chamane. Enfin, les trois espèces ont généralement leurs représentants dessinés dans le bas du tambour. Le chant glisse ainsi d'une description explicite du costume («les bandelettes de mon costume») à une référence possible aux dessins du tambour et aux murs de la yourte, mobilisant ainsi trois espaces de référence immédiats.

Mon esprit téléoute, âme du troupeau,

Ours brun, qui regarde à travers un anneau,

Mes esprits oiseaux [hus-tös], âme de l'homme;

À la tête du meuble de tête [pas paraan],

Âme de la tête du petit enfant,

Grande mère Ymaj,

Des coquillages blancs, du bouton de bronze,

Tu tires du fil de soie rouge.

Cette fois, la yourte est explicitement mobilisée comme espace de référence immédiat. L'esprit téléoute, absent des costumes et des tambours chamaniques, a son amulette dans la partie nord de la yourte. Cet animal cité ensuite est ici clairement celui de la yourte: «l'anneau » à travers lequel il regarde fait partie des éléments dont est constituée son amulette. L'ours apparaît donc à deux reprises, une première fois dans l'espace de référence du tambour en compagnie des serpents et des grenouilles, une seconde fois sous son aspect d'amulette dans la yourte. Avec ensuite Ymaj, localisée explicitement par le chant «à la tête du meuble de tête», c'està-dire dans les environs du coin d'honneur, on se situe indubitablement dans la yourte. Le chant se poursuit ainsi:

Créé par le khan chinois,

Venu de la célèbre Touva,

Arrivé du mont Sabyna,

Toi, dont les flèches ne tombent jamais à terre, 
Tu tires sans rater, Seigneur Gaucher.

Sorti de la noire Mongolie,

Attaché au pieu d'acier au centre de la terre,

Tuma le Noir au visage plus noir que la terre.

Légumes de la terre noire,

Étoiles du grand ciel,

En montant ouvrez la route.

Dans ce passage, le chamane nomme des cavaliers que nous savons situés dans la partie gauche du tambour et le coin sud de la yourte, deux espaces de référence immédiats possibles. Les trois derniers vers suggèrent un mouvement de «montée» qui va de la terre noire vers le ciel.

À ce moment, la source indique que, «ayant tiré tous les tös [esprits] de la porte au lieu d'honneur, s'inclinant vers le feu, il chante la mère Feu ». Le chamane a donc accompli physiquement dans la yourte un mouvement de l'est vers l'ouest. Se tenant maintenant dans le coin d'honneur (tör) face au foyer, il entonne une louange de l'esprit du feu, suivie de l'invocation: «Que les Trois Biches [Orion] donnent le bonheur!» L'assistance des profanes lui répond: "Oui, qu'il en soit ainsi. Que les ciels [kudajlar] nous le donnent. Qu'ils ne brisent pas l'âme. » L'espace de référence de cet échange de prières est le ciel, représenté dans la yourte par l'autel du ciel au pied duquel se tient maintenant le chamane et, dans le tambour, par la partie la plus haute où les trois biches d'Orion sont souvent figurées.

Si l'on résume la progression accomplie par le chant, on voit que l'on est passé des êtres les plus bas de la partie «basse» (nord), comme l'ours, à l'être le plus élevé de la partie «basse», Ymaj, avant d'évoquer les cavaliers appartenant à la partie «haute» (sud) pour accéder jusqu'aux entités célestes, les plus éminentes de la partie «haute». Cette ascension en paroles est en concordance avec le déplacement réel accompli par le chamane depuis la porte jusqu'au tör.

Le chamane fait ensuite trois fois le tour du foyer, ce qui indique un changement de scène. On exécute des libations tandis que le chant invoque les esprits des montagnes environnantes. Le chamane obtient d'eux la restitution de l'âme du malade et fait le geste de l'installer dans son tambour. Il conduit alors le patient près de la porte et prononce ces paroles:

Devenu une grenouille, bondis!

Devenu un serpent, rampe!

Ne sois plus un démon [ajna] qui ne se sépare pas et ne part pas,

Ne sois plus un diable [irlik] toujours lancinant,

Que la tête du démon soit en bas,

Que la tête de l'humain lunaire soit en haut,

Que les invisibles soient en bas,

Que les humains solaires ${ }^{3}$ soient en haut.

L'espace de référence immédiat est certes la porte de la yourte mais aussi le bas du tambour car, à la différence du serpent, la grenouille figure seulement sur l'instrument. Les paroles du chant s'adressent d'abord à un
3. Les humains sont qualifiés de «lunaires" et "solaires " dans les chants chamaniques, par opposition aux habitants du monde inférieur qui n’a ni lune ni soleil. 


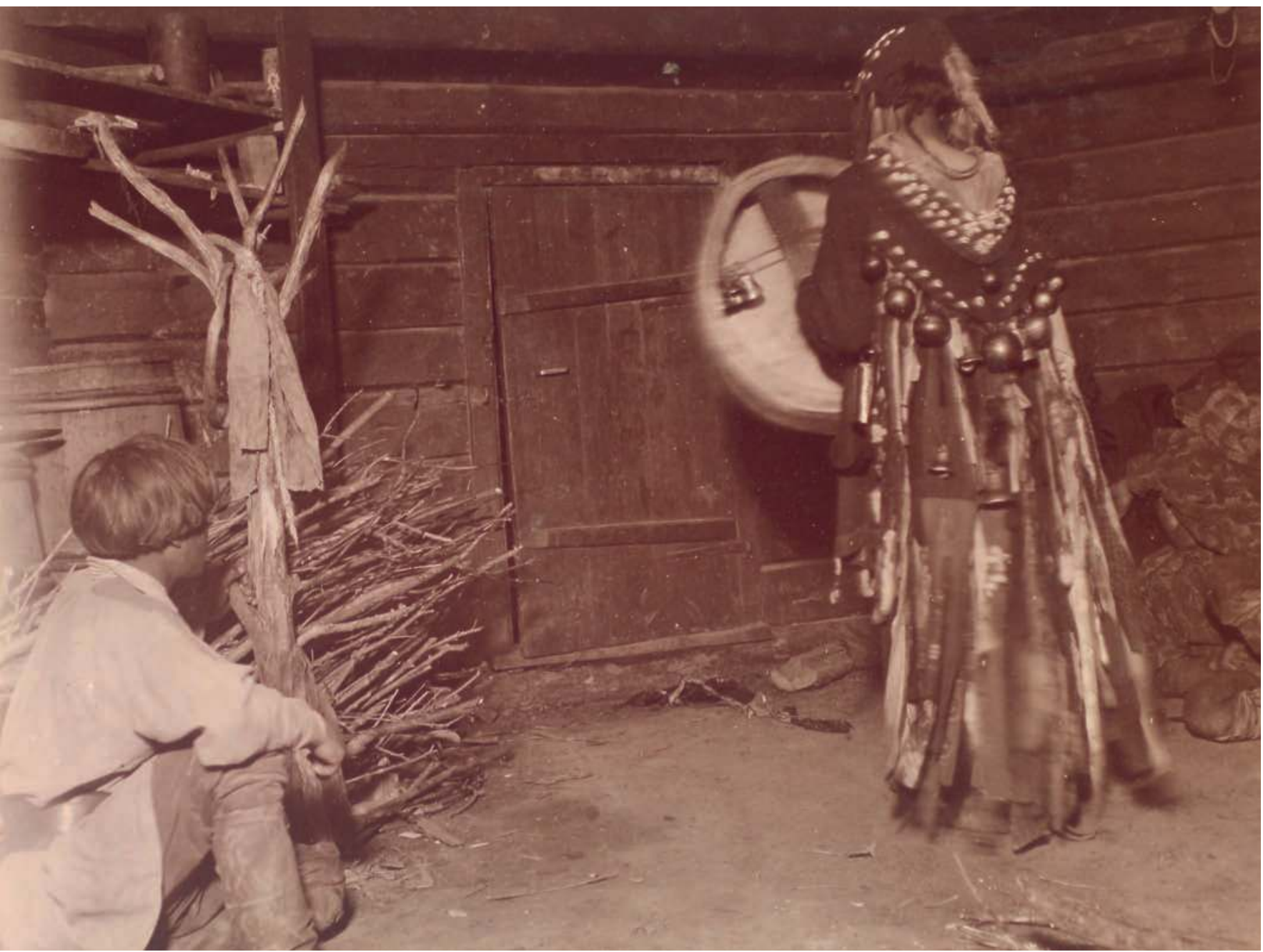

fig. 9

Ø.M. Olsen, Rituel dans

une yourte khakasse, 1914

Le chamane se tient face

à la porte, dans la partie est

de la yourte. Bibliothèque

nationale de Norvège. 


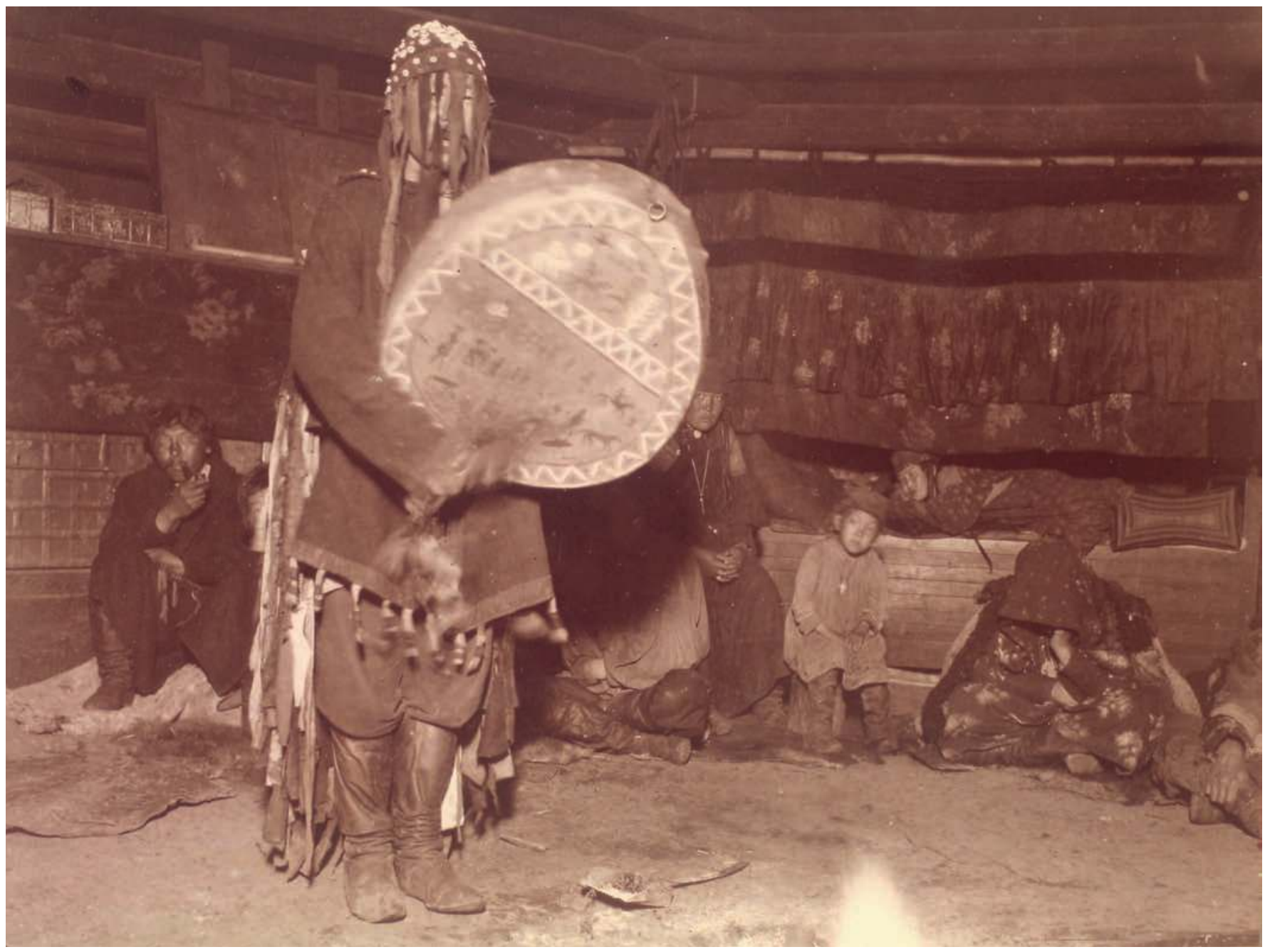

fig. 10

Ø.M. Olsen, Rituel dans

une yourte khakasse, 1914.

Le chamane se tient dans

la partie d'honneur devant

l'autel et face au feu,

dans le coin sud-ouest

de la yourte. Bibliothèque

nationale de Norvège. 
destinataire ambigu qui est à la fois le patient et le démon pathogène fixé en lui. Puis les deux entités sont incitées à se dissocier, l'officiant exigeant du démon qu'il reparte vers le monde inférieur qu'il n'aurait pas dû quitter ("Que la tête du démon soit en bas"). En même temps, il doit faire remonter le malade vers le monde du milieu ("Que la tête de l'humain soit en haut»).

Le chant continue avec une invocation des étoiles, c'est-à-dire d'entités célestes à l'opposé diamétral de l'ours et du serpent précédemment cités. Ce bond du plus bas au plus haut est suivi d'une redescente à travers les amulettes de la yourte. Le chamane nomme Ymaj «à la tête du meuble de tête", passant donc du sud-ouest à l'ouest-nord-ouest, puis les esprits médians, "la femme esprit téléoute au nord [altynzaryh tileg tös]" et face à elle l'«esprit oiseau au sud [ustünzaryh hus tös]", et la descente s'achève près de la porte avec l'ours au «vieil anneau ».

Après cet aller et retour en paroles du plus bas au plus haut de la yourte, le chamane accomplit l'action capitale de la restitution de l'âme (hut). II la représente concrètement en donnant à boire au patient du lait dans lequel il a «versé» l'âme. Invoquant les ciels créateurs, l'officiant fait alors accomplir au malade le même parcours de la porte vers l'autel qu'il a lui-même exécuté au début du rituel, passant de l'obscurité inférieure de l'ours à la clarté supérieure de l'autel.

La technique de cure menée par le chamane Pituk passe donc par la création d'un espace ordonné complexe, riche d'évocations, qui transforme en itinéraire les listes de noms d'esprit des séquences chantées et sert de cadre à des déplacements dont le but est de rétablir une topologie cosmique perturbée. Les quelques pas du patient le menant de la porte aux environs de l'autel se donnent ainsi à percevoir comme une remontée depuis le monde d'en bas vers le monde du milieu. Simultanément, les présences négatives qui s'étaient installées dans son corps sont abandonnées près de l'ours qui est chargé de les maintenir à leur place.

\section{Espace virtuel et espace réel}

Un rituel chamanique accomplit une intégration entre deux types d'interaction: une interaction ordinaire entre le chamane et ses patients et une interaction ésotérique entre le chamane et ses esprits (Hanks 2009). Dans les traditions sibériennes, cette dynamique interactionnelle se double d'une coordination entre les cadres spatiaux de ces interactions, la scène rituelle et les paysages du "voyage". Le chamane doit amener les nonchamanes à partager avec lui non pas son expérience, qui est supposée lui être propre car liée à ses capacités perceptives exceptionnelles, mais l'organisation spatiotemporelle qui sert de cadre à cette expérience mise en scène.

Le "voyage chamanique", loin d'être réductible à une expérience extatique intime, procède par un enrichissement progressif de l'espace réel où se situe l'assistance afin de faire émerger un cadre spatial modifié. Au début du rituel khakasse, le chant du chamane énumère des références à des esprits associés à des paysages lointains et inaccessibles pour le public - steppes mongoles, ciel, monde souterrain - qui constituent un 
espace de référence distant, ou espace virtuel. Ces entités sont figurées dans l'espace réel de la scène rituelle par des projections indicielles sur les diverses surfaces des espaces de référence immédiats: le costume chamanique, le tambour, les murs de la yourte. Ces points de connexion sont activés au fil du chant, qui glisse entre les espaces de référence et les fait se chevaucher dans des relations ambiguës d'identification et de projection.

Le tambour peint, cercle vertical orienté, fournit le modèle topologique permettant de penser la projection de l'ordre cosmique vertical dans la latéralité et la verticalité du corps du chamane, mais aussi dans l'horizontalité orientée de la yourte. Une fois ce principe de projection mis en place par les coordinations que le chant active, la yourte est donnée à penser comme un espace fusionné et multiple. Dans ce cadre nouveau, les mouvements et les gestes des participants au rituel se chargent d'une résonance spatiale d'une grande richesse. Chaque déplacement à l'intérieur du plan horizontal de la yourte dessine en même temps un trajet dans la verticalité virtuelle qui lui est coordonnée. Lorsque le chamane s'avance de la porte vers l'autel, on comprend qu'il s'élève vers un niveau supérieur du monde et qu'il élève avec lui le patient. Lorsqu'il revient près de la porte, on le voit redescendre vers la terre.

On saisit mieux maintenant en quoi les dessins peuvent aider les chamanes à «s'orienter». Si l'espace virtuel, la yourte et le corps du chamane se nouent dans le schéma spatial du tambour, l'officiant peut facilement se repérer dans l'univers par de simples gestes dans la yourte. À partir de sa proprioception, de la sensation de ses deux mains, il peut faire dériver des asymétries entre gauche et droite, stabilité et mouvement, arbres et cavaliers, taïga et steppe, et les différentes routes qui en dérivent. II doit mémoriser ses dessins non pas seulement visuellement mais de façon synesthésique en les couplant à la perception de ses fonctions motrices. Les dessins chamaniques sont en définitive moins des supports expressifs qu'une technologie subtile et efficace contribuant à transmettre et stabiliser des modèles de coordination du corps et de l'espace au cœur de la scène rituelle.

\section{remerciements}

Cette étude est née de discussions stimulantes avec Carlo Severi, à qui je suis heureux d'exprimer ici ma gratitude pour ses conseils et son soutien. Je remercie également Andrea Luz Gutierrez Choquevilca pour ses précieuses remarques et suggestions. Les données présentées ici s'appuient notamment sur des recherches dans les archives du musée de Minoussinsk en mars 2011 et dans celles de la Kunstkamera de Saint-Pétersbourg en novembre 2011 réalisées grâce à un financement dans le cadre du projet de recherche ANR "Anthropologie de l'art: création, rituel, mémoire». 


\section{Adrianov, Aleksandr Vasil'evič}

1909 «Ajran v žizni minusinskogo inorodca ", in A.D. Rudnev (éd.) Sbornik v čest' semidesjatiletija G.N. Potanina. Saint-Pétersbourg, Tip. V.F. Kiršbaum: 489-524.

\section{Anohin, Andrej}

1924 «Materialy po šamanstvu Altajcev", Sbornik muzeja antropologii i ètnografii 4(2) : 1-148.

\section{Basilov, Vladimir}

1984 Izbanniki duhov.

Moscou, Politizdat.

\section{Berthoz, Alain}

1997 Le Sens du mouvement. Paris, Odile Jacob.

\section{Butanaev, Viktor}

2003 Burhanizm tjurkov Sajano-Altaja. Abakan, Izdatel'stvo hakasskogo gosudarstvennogo universtita.

2006 Tradicionnyj šamanizm Hongoraja. Abakan, Izdatel'stvo hakasskogo gosudarstvennogo universtita.

\section{Diószegi, Vilmos}

1998 (1978) «Pre-islamic shamanism of the Baraba Turks and some ethnogenetic conclusions ", in Mihaly Hoppál (éd.), Shamanism. Selected writting of Vilmos Diószegi. Budapest, Akadémiai Kiadó : 228-298.

\section{Gmelin, Johann Georg}

1751-1752 Reise durch Sibirien von dem Jahr 1733-1743. Göttingen, A. Vandenhoecks, 4 vol

\section{Hanks, William F.}

2009 "Comment établir un terrain d'entente dans un rituel?",

in Julien Bonhomme et Carlo Severi (éd.), Paroles en actes. Paris,

L'Herne ("Cahiers d'anthropologie sociale») : 87-113.

\section{Ivanov, Sergej Vasil'evič}

1954 Materialy po izobrazitel'nomu iskusstvu narodov Sibiri xıx-načala

$x x v$. Moscou-Leningrad, Izdatel'stvo akademii nauk SSSR.

1955 «K voprosu o značenii izobrazenij na starinyh predmetah kul'ta u narodov Sajano-Altajskogo nagor'ja ", Sbornik muzeja antropologii i ètnografii 16: 165-265.

1979 Skul'ptura altajcev, hakasov i sibirskih tatar. xvIII-pervaja četvert' $x x v$. Leningrad, Nauka.

\section{Jakovlev, Evgenij Konstantinovič}

1900 Ètnografičeskij obzor inorodčeskogo naselenija doliny Južnogo Eniseja i ob'jasnit. katalog ètnogr. otd. Muzeja. Minoussinsk, Tipografija V.I. Kornakova.

\section{Katanov, Nikolaj Fedorovič}

1897 Otčet o poezdke soveršennoj s 15 maja po 1 sent. 1896 goda v Minusinskij Okrug Enisejskoj Gubernii. Kazan, Tipo-lit. Imp. Kazansk. Univ.

1907 Narečija urjanhajcev (sojotov), abakanskih tatar i karagasov. I, Teksty. II, Perevody. Saint-Pétersbourg, Imprimerie de l'Académie des sciences.

$\mathbf{2 0 0 0}$ Izbrannye naučnye trudy. Ankara, Türksoy.

\section{Klemenc, Dmitrij}

1890 «Neskol'ko obrazcov bubnov minusinskih inorodcev ", Zapiski VSOIRGO po ètnografii 2(2) : 25-35.

\section{Kyzlasov, Leonid Romanovič et Leont'ev, N.V.}

1980 Nardonye risunki hakasov. Moscou, Nauka.

\section{Lot-Falck, Éveline}

1961 «À propos d'un tambour de chaman toungouse", L'Homme 1(2): 23-50.

\section{Lot-Falck, Éveline et Diószegi, Vilmos}

1973 "Les tambours chamaniques des Turcs barabin : étude comparée ", L'Ethnographie 67 : 18-46.

\section{Oppitz, Michael}

2007 Trommeln der Schamanen. Zurich, Völkerkundemuseum der Universität Zürich.

\section{Potapov, Leonid Pavlovič}

1981 «Šamanskij buben kačincev kak unikal'nyj predmet ètnografičeskih kollekcij ", Sbornik muzeja antropologii i ètnografii 36: 125-137.

1991 Altajskij šamanizm. Leningrad, Nauka.

\section{Radloff, Wilhelm}

1884 Aus Sibirien. Lose Blätter aus meinem Tagebuche. Leipzig, Weigel, 2 vol.

\section{Severi, Carlo}

2007 Le Principe de la chimère: une anthropologie de la mémoire. Paris, Rue d'Ulm-musée du quai Branly.

2011 «L'espace chimérique. Perception et projection dans les actes de regard", Gradhiva 13: 9-47.

\section{Stépanoff, Charles}

2009 "Le chamane-koulak, la dernière métamorphose. Le chamane dans le cinéma soviétique des années 1920-1930", in Élisabeth Anstett-Gessat (éd.), Un patrimoine sous influences. Paris, Pétra: 113-141.

\section{Tadina, Nadežda}

\section{Alekseevna}

2006 «Semantika vnutrennego prostranstva žilišča i ètiketnye predpisanija u altajcev », Vestnik tomskogo gosudarstvennogo universiteta, 60: 122-126; http://new.hist.asu.ru/naltai/ ualtaiseman.html.

\section{Warnier, Jean-Pierre}

1999 Construire la culture matérielle. L'homme qui pensait avec ses doigts. Paris, PUF. page 144 et ci-contre Ø.M. Olsen, Rituel dans une yourte khakasse, 1914. Le chamane se tient

dans la partie d'honneur devant

l'autel et face au feu, dans le coin sud-ouest de la yourte. Bibliothèque nationale de Norvège. 


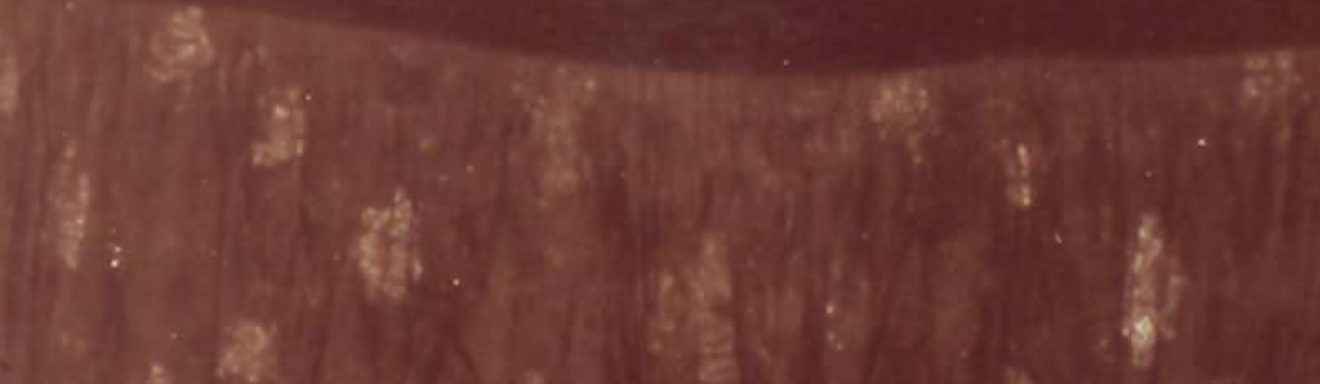

4.

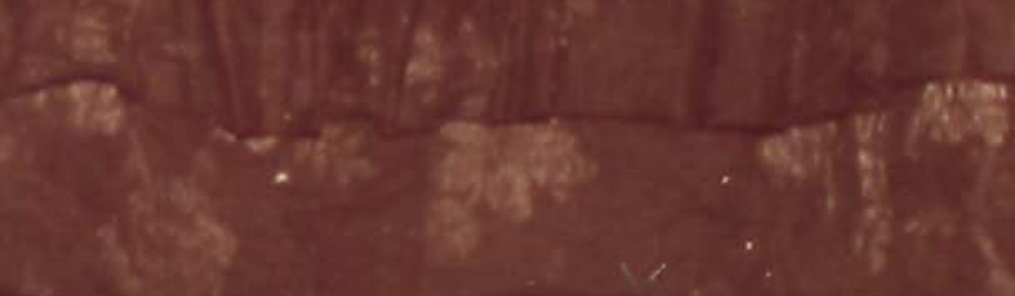

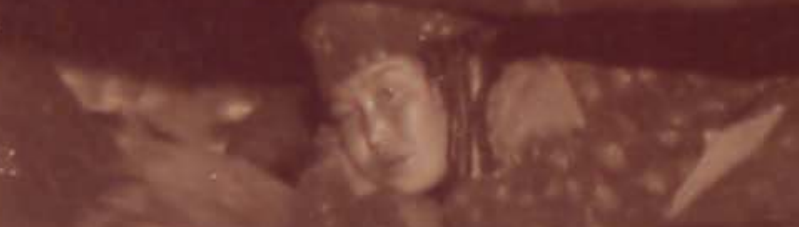

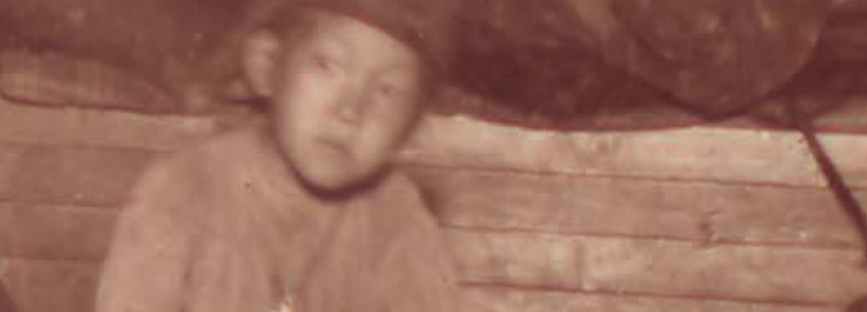

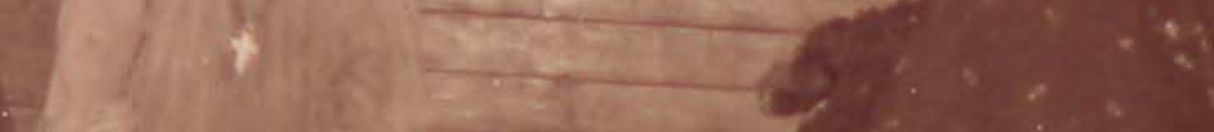

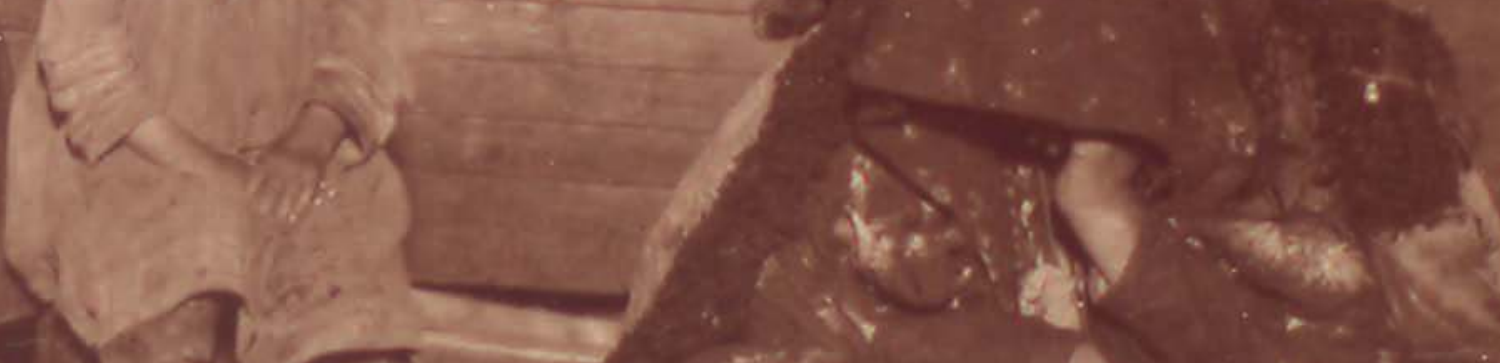

$\mathrm{H}^{1}$ $\rightarrow$ it a

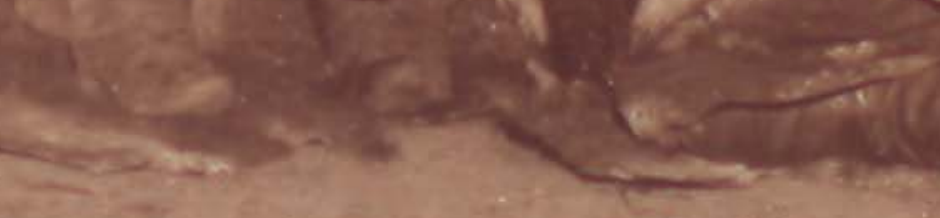

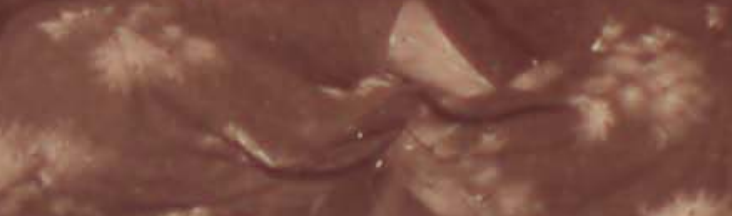

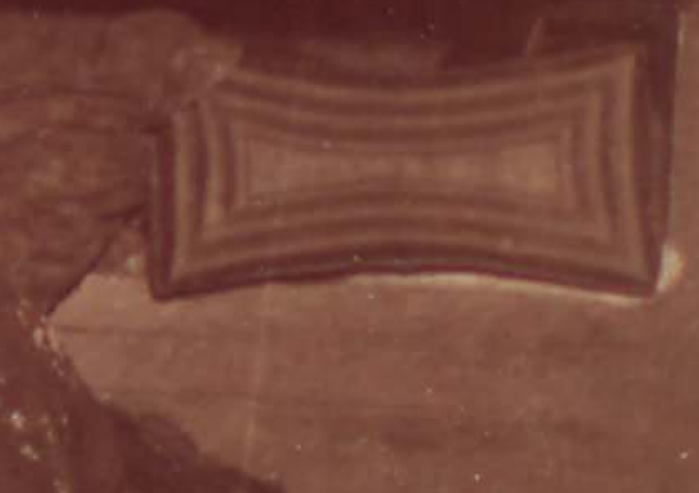

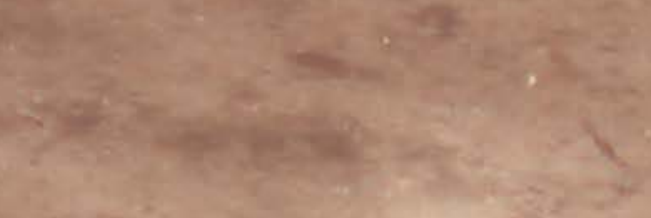
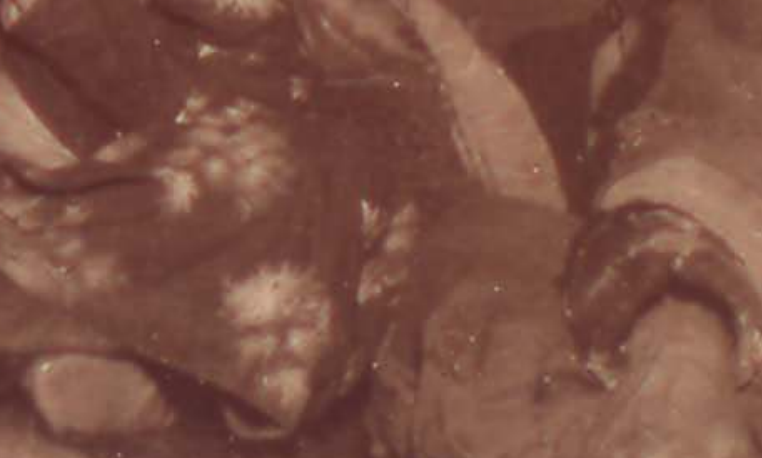

be

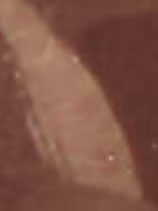

